\title{
Image Processing Techniques as a Support to Transmitter Positioning Determination in Cognitive Radio Networks
}

\author{
Liliana Bolea, Jordi Pérez-Romero, Ramón Agustí, Oriol Sallent \\ Universitat Politècnica de Catalunya (UPC) \\ c/ Jordi Girona, 1-3, 08034, Barcelona, Spain \\ \{lilianab, jorperez, ramon, sallent $\} @$ tsc.upc.edu
}

\begin{abstract}
Cognitive radio is a new paradigm for wireless communications offering a solution to conciliate the current spectrum demand growth and underutilization without changes to the existing legacy wireless systems. Secondary users should be able to identify spatial and temporal spectrum holes not occupied by primary users and use them opportunistically, without generating interference to primary receivers. For that purpose, having knowledge of the primary network is required to ensure an appropriate secondary user operation. In this context, and assuming there is no cooperation between primary and secondary networks, this paper proposes a new framework, based on image processing techniques, aimed at combining a number of sensed samples at different geographical positions collected by secondary sensors, in order to estimate the positions of the different primary transmitters. The results can be used to discover frequencies that can be used by a secondary network without disturbing primary receivers. Our results, performed in a realistic scenario, show the efficacy of the proposed framework in estimating transmitter positions.
\end{abstract}

Keywords: Sensing, Secondary Spectrum Use, Radio Environment Map, Image Processing.

\section{INTRODUCTION}

Wireless technologies are rapidly evolving to allow operators to deliver more advanced multimedia services. For that, the need of spectrum is growing every day. However, some recent spectrum measurements have demonstrated that the licensed part of the radio spectrum is poorly utilized [12]. In this context, Cognitive Radio (CR) and dynamic spectrum access paradigms have emerged as a promising solution to conciliate the current spectrum demand growth and its underutilization without changes to the existing legacy wireless systems [3]. CR enables much higher spectrum efficiency through opportunistic spectrum access. Therefore, it is an attractive technology for future wireless communications.

The basic idea of $\mathrm{CR}$ is to allow secondary/unlicensed users (SUs) to access in an opportunistic and non-interfering manner some licensed bands temporarily unoccupied by primary/licensed users (PUs). The operating principle is to identify spatial and temporal spectrum gaps not occupied by PUs, place SU transmissions within such spaces and vacate the channel as soon as PUs return.

The term "spectrum holes" stands for those licensed subbands of the radio spectrum that are not actually utilized at a particular instant of time and specific geographic location [4]. These spectrum holes could be used opportunistically by (SUs) provided that they are able to sense the spectrum to detect the presence of either PU or SU transmissions and to adapt to the varying spectrum conditions, ensuring that the primary rights are preserved [5].

The primary-secondary spectrum sharing can take the form of cooperation or coexistence. Cooperation means there is explicit communication and coordination between primary and secondary systems, and coexistence means there is none [6]. When sharing is based on coexistence, secondary devices are essentially invisible to the primary. Thus, all of the complexity of sharing is borne by the secondary and no changes to the primary system are needed.

Under the above consideration, assuming the coexistence case, a key enabler of the cognitive radio technology is the detection of the spectrum holes through spectrum sensing. As SUs may not have accurate knowledge about the whole spectrum at all times, in order to avoid generating interference, techniques for detecting PUs need to be employed before spectrum access. Several techniques for detecting primary transmitters through matched filter detection, energy detection, and cyclostationary feature detection have been investigated by related work [7-12].

Multipath fading and shadowing, the fundamental characteristics of wireless channels, present a major challenge of spectrum sensing. In [13], it has been shown that the detection capability of the CR network can be improved through cooperation, by letting multiple CR users sense the channel instead of designating a fixed CR user for spectrum sensing. Then, cooperative spectrum sensing [1316] refers to sensing methods where local sensors sense and then send information to the centre where the final decision on PU presence is made.

Knowing that cooperative strategies can make good use of network resources, obtain higher gain and make the network steadier, we assume the cooperation principle as a technique to improve the performance of cognitive secondary networks. In this respect, this paper proposes a new methodology, based on image processing techniques, aimed at combining a number of sensed samples at different geographical positions collected by secondary sensors, in order to estimate the positions of the different primary transmitters and to have an insight about their coverage area. The proposed methodology could be used to build databases containing the relevant aspects of radio environment characterization, such as the so-called Radio Environment Map (REM) in [17]. REM can also be viewed as the 
generalization of the available resource map proposed by Krenik for cognitive radio applications in unlicensed wide area networks [18-19]. Similarly, in [20], the sensorial radio bubble concept is presented using a spectrum map to represent the context in a cognitive radio network. Our previous work in [21] also introduced the image processing to identify primary transmitters positions, in a theoretical scenario. This paper extends the previous work by considering a realistic scenario and comparing two different proposed methods to estimate the primary transmitters' positions.

The rest of the paper is organized as follows. Section II presents in more detail the system model and problem that is considered in this paper, while Section III describes the proposed methodologies. Section IV presents performance results under different conditions. Finally, conclusions are presented in Section $\mathrm{V}$.

\section{System Model AND PROBLEM DESCRIPTION}

Let assume a generic scenario such as the one depicted in Fig. 1. It is characterized by a number of transmitters corresponding to different Radio Access Technologies (RATs) which operate at different frequencies and having very different coverage areas (e.g. the central transmitter operating in a broadcast-like RAT with an extensive coverage area at frequency $f_{5}$, or the transmitters operating at RATs 1 and 2 with frequencies $f_{1}, f_{2}, f_{3}$ and $f_{4}$ that could correspond to some cellular-like RATs).

The secondary network can rely on the information measured by a number of sensors randomly scattered in the scenario and that could be built-in e.g. mobile terminals, and the appropriate post-processing of this information, which is the focus of this paper. We assume that the sensors cooperate with each other in a centralized manner, where a central entity plays the role to gather all sensing information from the sensors and to detect the positions and coverage areas of the primary transmitters.

A sensor measures the received power in a number of $\mathrm{N}$ specific frequencies in its geographical position. It is assumed that frequency $f_{i}(i=1,2, \ldots N)$ is detected by the sensor at position $(\mathrm{x}, \mathrm{y})$ when the received power is above a given threshold $\mathrm{P}_{\text {th }}\left(\mathrm{f}_{\mathrm{i}}\right)$. We present two different methods, denoted here as binary and multi-level methods. In the binary

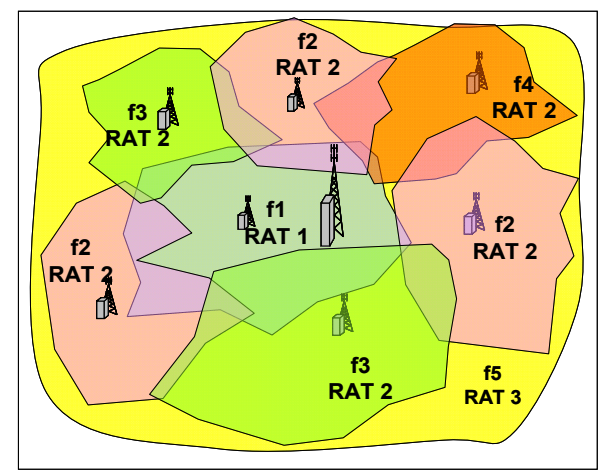

Figure 1. Generic scenario with different RATs and frequencies. method, each sensor sends just one bit, for each frequency $f_{i}$, to a central entity, in charge of combining the measurements of every sensor. Instead, in the multi-level method, the value detected by a given sensor for each frequency is quantified to a set of $2^{k}$ values. Then, the sensor will send to the central entity this value encoded as a word of $k$ bits.

The problem considered here consists in defining a methodology to smartly combine the different measurements at random positions, which represent a partial vision of the scenario, in order to get a full vision in which the positions and coverage areas of the different primary transmitters are estimated. It is worth mentioning that the considerations on the sensing process itself (such as errors in the process or the determination on which frequencies has to sense every sensor) and the means to report the sensing results are out of the scope of the paper and are left for future work.

\section{Proposed Methodologies}

The proposed methodologies assume that the radio environment can be characterized by an image, where each pixel (i.e. a rectangular area of dimensions $\Delta x \times \Delta y$ ) contains the information about the frequencies that are measured in this area. This information can be encoded either with the binary or the multi-level methods. Then, given that only the values of the pixels where a sensor is located are known, these values will be combined using image processing techniques in order to reconstruct the overall image and to discover the transmitter positions, as it is illustrated in Fig. 2. In the following, the two proposed methods are presented. In all cases it is assumed that a pixel can only have the result of one sensor.

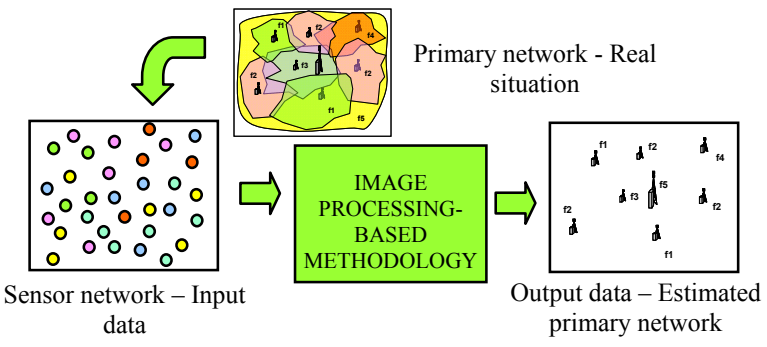

Figure 2. Inputs and outputs of the considered problem.

\section{A. Binary method}

As we mention in Section II, in this method, each sensor measures the received power in a number of specific frequencies in its position and sends just one bit, for each frequency $f_{i}(i=1,2, \ldots N)$, which takes the value 1 if the received power is above a given threshold $\mathrm{P}_{\text {th }}\left(\mathrm{f}_{\mathrm{i}}\right)$ and 0 otherwise. It can be obtained the following binary representation for each frequency at each sensor position:

$M\left(f_{i}, x, y\right)=\left\{\begin{array}{rr}1 & \text { if } f_{i} \text { detected in }(x, y) \Leftrightarrow \text { if } P\left(f_{i}, x, y\right) \geq P_{t h}\left(f_{i}\right) \\ 0 \quad \text { if } f_{i} \text { not detected in }(x, y) \Leftrightarrow \text { if } P\left(f_{i}, x, y\right)<P_{t h}\left(f_{i}\right)\end{array}\right.$

From this binary representation, it is possible to characterize the measurement at all frequencies given by the sensor at coordinate $(\mathrm{x}, \mathrm{y})$ by a value corresponding to the 
sum of the binary representations of all the $\mathrm{N}$ considered frequencies:

$$
I(x, y)=\sum_{i=1}^{N} M\left(f_{i}, x, y\right) 2^{i-1}
$$

We assume that the coverage area of a transmitter to be discovered will be approximately circular, which would be valid in terms of average received power according to the distance-dependent path loss whenever omnidirectional antennas are used. Then, the proposed methodology aims at identifying in the image the existing circular regions, using an object-based reconstruction technique.

The steps of this method are illustrated in Fig. 3 and explained in the following.

- First, from the information received from the secondary sensors we build an image by interpolating the intermediate pixels for those positions where no sensor was available. We do that through the nearest neighbour interpolation technique, by attributing to each unknown pixel the value of the nearest known pixel.

- From the resulting image, we build a set of $\mathrm{N}$ binary images, each one corresponding to one frequency. The pixels of the binary image corresponding to frequency $f_{i}$ take the binary values $M\left(f_{i}, x, y\right)$. These binary images will be used as the basis to identify the different "objects" (i.e. an object is a region where a certain frequency $f_{i}$ is detected, or correspondingly where the pixels of the binary image take the value 1).

- It is possible that in some cases, some objects are not properly detected, because they are not clearly separated with each other. In order to eliminate this drawback, before of object-based reconstruction technique, we apply an image processing technique called erosion to the binary images resulting from the interpolated image. In the erosion, the value of the output pixel is the minimum value of all the pixels in the input pixel's neighborhood. We assume that a pixel's neighborhood corresponds to a circular structuring element [22], defined by a circular area of radius 5 pixels around the input pixel. Note that in the particular case of a binary image, if any of the pixels of the neighborhood is set to the value 0 , the output pixel after the erosion will be set to 0 , which will tend to decrease the size of the objects and thus to separate them.

- Then, for each binary image (i.e. for each frequency $\mathrm{f}_{\mathrm{i}}$ ), we apply an object-based reconstruction technique in order to detect the objects and measure their properties. Object detection is done following the so-called connected-component labelling technique [23] that consists in scanning the image and making groups of adjacent pixels having the same value (it is assumed that pixels are adjacent if their edges touch). For each detected object, the measured properties are the centroid and the radius, which correspond, respectively, to the centre and radius of a circle with the same area than the object. With these properties, the object-based reconstruction process regenerates a new image replacing each object by a circle with the corresponding radius and centered in the corresponding centroid.

- Because of the prior erosion process, the resulting object area after object-based reconstruction technique has become smaller than in the binary images, which would lead to more reduced coverage areas than in the real situation. To compensate this effect, we apply the dilation technique to the binary images resulting from the object-based reconstruction technique. The dilation is the image processing technique opposite to the erosion process, and in this case the value of the output pixel is the maximum value of all the pixels in the input pixel's neighborhood. The dilation is the image processing technique opposite to the erosion process, and in this case the value of the output pixel is the maximum value of all the pixels in the input pixel's neighborhood [22]. In particular, in a binary image, if any of the pixels of the neighborhood is set to the value 1 , the output pixel is set to 1 , which will tend to increase the size of the objects. The same neighborhood shape (i.e. circular structuring element) as in the erosion is considered. After dilation, a second object based reconstruction process is carried out, to obtain the final centroids and radii of the detected objects. Note that the computed centroids will correspond to the final estimated transmitter positions. In addition, the radii will provide a first insight of the coverage ranges.

- Due to the shadowing effects in the propagation, after the reconstruction process, it may happen that certain objects are detected with an area significantly smaller than that of the rest of objects, so they cannot be considered as transmitters. To cope with this, in the last step, we filter out the resulting images by eliminating those objects that have an area below a fraction $\alpha$ of the average area of all the detected objects.

- Finally, after this filtering, we combine the binary images to obtain a new image including information of all the frequencies. This image includes the transmitter estimated positions, coverage areas assumed to be circular and frequencies of the different primary transmitters.

\section{B. Multi-level method}

In the multi-level method, each sensor measures the received power in a number of specific frequencies in its position and quantifies this value in a set of $2^{k}$ levels with quantification step $\Delta$ and minimum power level $\mathrm{P}_{\text {th }}\left(\mathrm{f}_{\mathrm{i}}\right)$, as shown in Fig. 5. This value is sent to the central entity encoded as a word of $k$ bits, for each frequency.

From the information of each sensor, we build an image in which each pixel is characterized not only by the 


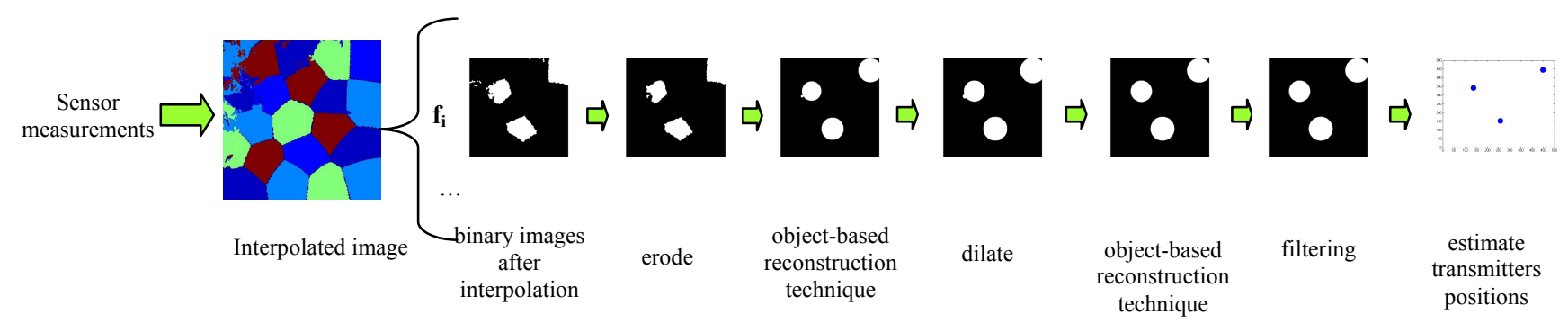

Figure 3. Steps of the binary method.

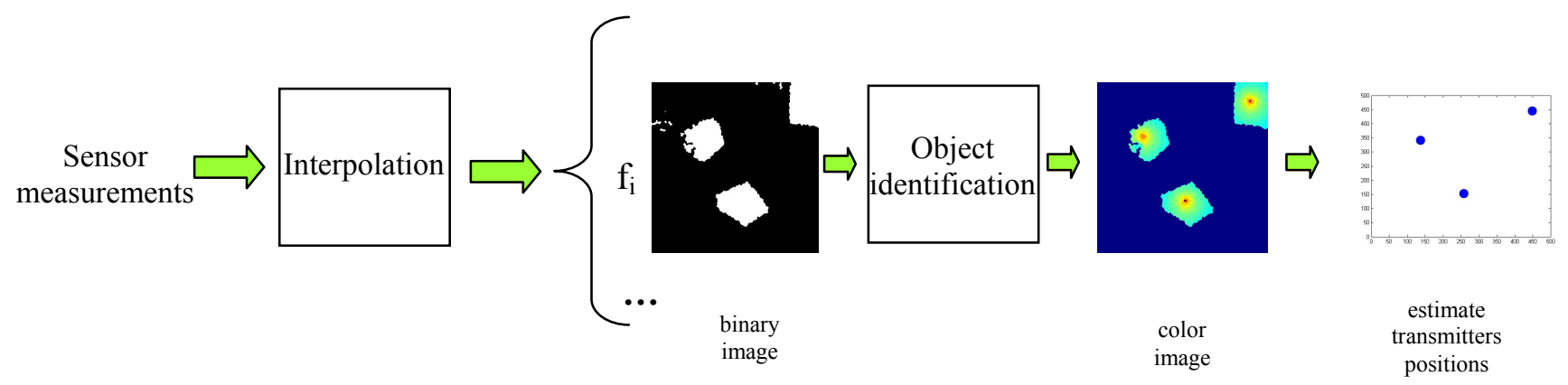

Figure 4. Steps of the multi-level method.

frequencies detected, like in the binary method, but also by the values of received powers at each frequency.

In particular, the value of one pixel will be a set of quantified values, one per frequency. The steps of multilevel method are shown in Fig. 4 and explained in the following.

- From the results of the sensors we build an image by interpolating the intermediate pixels for those positions where no sensor was available. The process is equivalent to the interpolation of the binary method, aiming to fill the empty spaces by attributing to each unknown pixel the value of the nearest known pixel

- From the resulting interpolated image, like in the binary method, we build a set of $\mathrm{N}$ binary images, one per frequency $f_{i}$, whose pixels take the value 1 when frequency $f_{i}$ is detected (i.e. it is above $\left.\mathrm{P}_{\text {th }}\left(\mathrm{f}_{\mathrm{i}}\right)\right)$ and 0 otherwise. These images will be used as the basis to identify the different "objects".

- Next, we apply object identification technique in order to detect the objects (i.e. regions where frequency $f_{i}$ is detected), following the same connected-component labelling technique explained in the description of the binary method. Furthermore, we remove those small objects that have an area below a fraction $\alpha$ of the average area of all the detected objects.

- In the next step, we convert the binary images into color images, using the quantified values from the received power at each frequency $f_{i}$ after the interpolation. Note in Fig. 4 that in the color image the same objects as in the binary image can be identified but now including the specific value of the received power in each pixel. Then, we analyze each object individually to determine the transmitter positions. In particular, given that the highest received power will be measured in the pixels where the primary transmitter is located, for each object we identify the pixels with the highest received power and calculate the centroid of these pixels. The value of the centroid will be the estimated transmitter position.

On the other hand, in addition to the transmitters' positions we can also obtain an estimate of the coverage range for each transmitter by applying to the binary images the same object-based reconstruction technique that was used in the binary method.

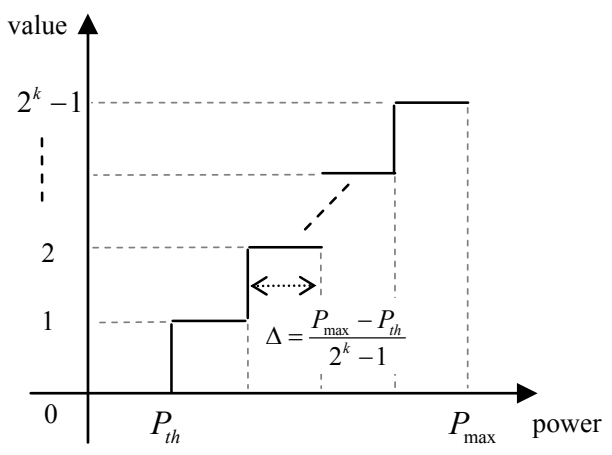

Figure 5. Quantization function

\section{RESULTS}

In order to illustrate the capabilities of the proposed methodology, it is evaluated in a cellular scenario with a 5 
frequency reuse pattern $\left(f_{1}, f_{2}, f_{3}, f_{4}, f_{5}\right)$. The total scenario size is $10 \mathrm{~km} \times 10 \mathrm{~km}$, the pixel size is $\Delta x=\Delta y=20 \mathrm{~m}$, and there are 21 primary transmitters. The transmitter power is $40 \mathrm{dBm}$, propagation losses are computed using a planning tool in a realistic environment. Power threshold $\mathrm{P}_{\text {th }}\left(\mathrm{f}_{\mathrm{i}}\right)$ is set to $-85 \mathrm{dBm}$ for all frequencies. Furthermore, in the multi-level method the number of bits is $k=5$ and the quantization step is $\Delta=3.6 \mathrm{~dB}$. In turn, the value of $\alpha$ in the object detection process is 0.3 .

In Fig. 6 we can see the original image representing the scenario with the position of transmitters, corresponding to a terrain in the surroundings of Barcelona, and in Fig. 7 we have the transmitters' coverage area where each color represents a different frequency.

A random sensor deployment with average density D sensors $/ \mathrm{km}^{2}$ is retained. As a first result, Fig. 8 plots the comparison between the real primary transmitter positions and the corresponding positions estimated with both methods. It can be observed that, although the two methods are able to identify quite accurately the positions, the estimation is more precise with the multi-level method.

In order to measure the accuracy in this estimation, we compute the relative error as the difference between the real transmitter position and the estimated position,

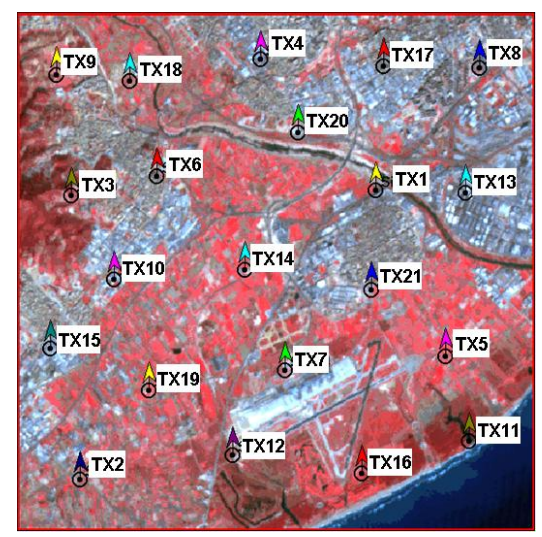

Figure 6. Image corresponding to real scenario and the transmitter positions

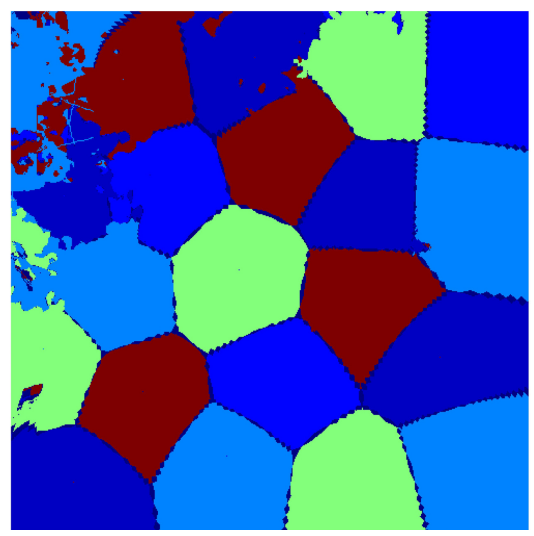

Figure 7. The coverage area of primary transmitters

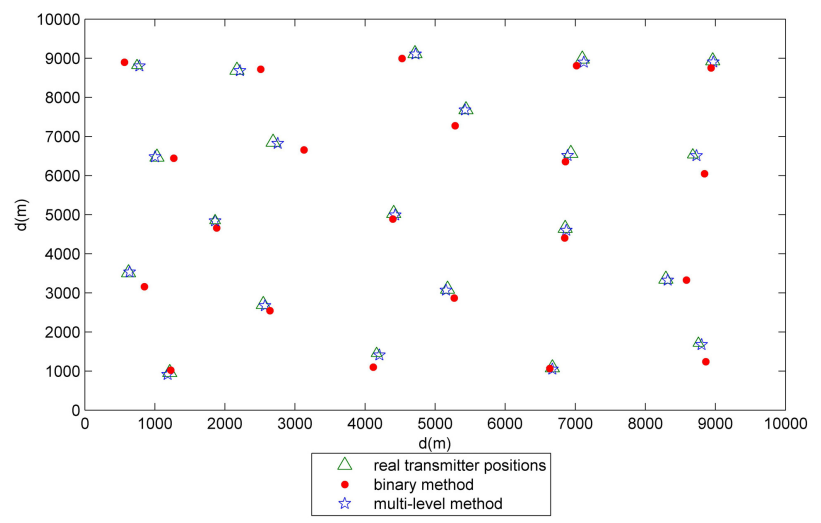

Figure 8. Real and estimated primary transmitter positions for $\mathrm{D}=100$ sensors $/ \mathrm{km}^{2}$.

divided by the estimated transmitter coverage radius. Fig. 9 represents the relative error for each of the 21 transmitters in the considered scenario for $\mathrm{D}=100$ sensors $/ \mathrm{km}^{2}$, together with the estimated radii with the two methods. It can be observed that, with the binary method, the values of the relative errors have a wide variation for the different transmitters and can be in some cases as high as $45 \%$. Instead, with the multi-level method, in all the cases the values of the relative errors are below $7 \%$. It can also be observed that the estimated radius for the different transmitters is quite similar for the two methods.

Fig. 10 shows the mean relative error and the standard deviation for different values of sensor density $\mathrm{D}$ with the two considered methods. For the binary method, notice that the mean error improves very slowly when increasing the sensor density. In particular, the mean relative errors are still above $24 \%$, for sensor densities as high as $D=100$ sensors $/ \mathrm{km}^{2}$. On the contrary, for the multi-level method, we can observe a more significant reduction in both the average error and the standard deviation as the density of sensors grows. Notice in this case that only for a rather low number of sensor densities (e.g. $\mathrm{D}=4$ sensors $/ \mathrm{km}^{2}$ ) the

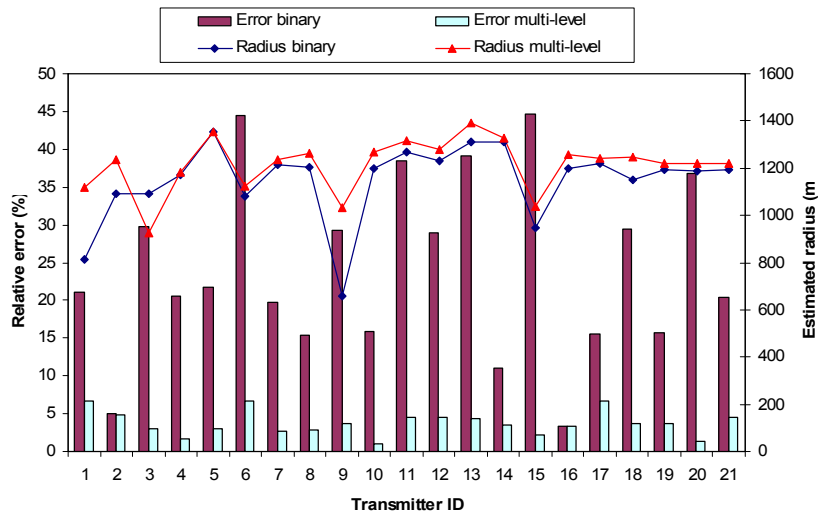

Figure 9. Relative error in transmitter positions and estimated radius for $\mathrm{D}=100$ sensors $/ \mathrm{km}^{2}$ with binary and multi-level methods. 


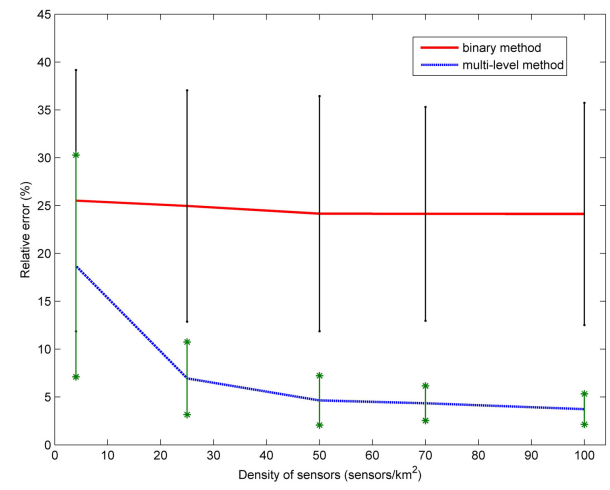

Figure 10. Mean relative error and standard deviation (represented as vertical lines) in the transmitter position estimation.

mean error as well as the standard deviation are high. These results allow establishing the minimum required density of sensors for a desired accuracy. For example, in order to achieve an error below $5 \%$, the required density of sensors will be $\mathrm{D}=50$ sensors $/ \mathrm{km}^{2}$.

\section{CONCLUSION}

In this paper we have proposed a novel framework based on image processing techniques, aimed at combining a number of sensed samples at different geographical positions collected by secondary sensors, in order to build a map with the estimated positions and coverage areas of the different primary transmitters. Utilization of this information in the context of secondary spectrum usage allows the secondary network to discover the presence of primary network transmitters and to use spectrum opportunities without causing interference to them. We applied two different methods on a real scenario: the binary and the multi-level methods. Our results with multi-level method reveal the advantages of the proposed framework, with relative errors below $7 \%$ in the transmitter position estimation.

\section{ACKNOWLEDGMENT}

This work has been supported by the Spanish Research Council and FEDER funds under COGNOS grant (ref. TEC 2007-60985). The support from the Spanish Ministry of Science and Innovation (MICINN) under FPU grant AP2008-02291 is hereby acknowledged.

\section{REFERENCES}

[1] T.X. Brown, "An Analysis of Unlicensed Device Operation in Licensed Broadcast Service Bands," in Proceedings of 1st IEEE DySPAN, Baltimore, MD, pp. 11-29, Nov. 2005.

[2] T. Kamakaris, M. Buddhikot, and R. Iyer, "A Case for Coordinated Dynamic Spectrum Access in Cellular Networks," in Proceedings of 1st IEEE DySPAN, Baltimore, MD, pp. 289-298, Nov. 2005.

[3] J. III Mitola and G.Q. Jr. Maguire, "Cognitive Radio: Making Software Radios More Personal," IEEE Pers. Commun., vol. 6, no. 4, pp. 13-18, Aug. 1999.

[4] S. Haykin, "Cognitive Radio: Brain-Empowered Wireless Communications," IEEE Journal on Selected Areas in Communications, vol. 23, no. 2, pp. 201-220, Feb. 2005.
[5] V. Petty et at, "Feasibility of Dynamic Spectrum Access in Underutilized Television Bands," in Proceedings of 2nd IEEE DySPAN, Dublin, pp. 331-339, Apr. 2007.

[6] J. M. Peha, "Emerging Technology and Spectrum Policy Reform," in Proceedings of ITU Workshop on Market Mechanisms for Spectrum Management, Geneva, Jan. 2007.

[7] A. Sahai, N. Hoven, and R. Tandra, "Some Fundamental Limits in Cognitive Radio," in Proc. of Allerton Conference on Communication, Control and Computing, Monticello, IL, Oct. 2004.

[8] I. Akyildiz, W.-Y. Lee, M. Vuran, and S. Mohanty, "Next Generation/Dynamic Spectrum Access/Cognitive Radio Wireless Networks: A Survey," Elsevier Computer Networks, vol. 50, no. 13, pp. 2127-2159, Sep. 2006.

[9] A. Fehske, J. Gaeddert, and J. Reed, "A New Approach to Signal Classification Using Spectral Correlation and Neural Networks," in Proceedings of 1st IEEE DySPAN, Baltimore, MD, pp. 144-150, Nov. 2005.

[10] J. Pérez-Romero, X. Gelabert, O. Sallent, and R. Agustí, “A Novel Framework for the Characterization of Dynamic Spectrum Access Scenarios," IEEE PIMRC Conference, Cannes, pp. 1-6, Sep. 2008.

[11] Tsai-Wei Wu and Hung-Yun Hsieh, "An Investigation of Primary Transmitter Detection Techniques in Cognitive Radio Networks from Network Optimization Perspective," IEEE WCNC Conference, Las Vegas, pp. 2753-2758, Apr. 2008.

[12] Biing Hwang Juang; G.Y. Li, and Jun Ma, "Signal Processing in Cognitive Radio," in Proceedings of the IEEE, vol. 97, no. 5, pp. 805-823, May 2009.

[13] A. Ghasemi and E. S. Sousa, "Collaborative Spectrum Sensing for Oppotunistic Access in Fading Environments," in Proceedings of 1st IEEE DySPAN, Baltimore, MD, pp. 131-136, Nov. 2005.

[14] G. Ganesan and Y.G. LI, "Cooperative Spectrum Sensing in Cognitive Radio - Part I: Two User Networks," IEEE Trans. Wireless Communications, vol. 6, no. 6, pp. 2204-2213, 2007.

[15] G. Ganesan and Y.G. LI, "Cooperative Spectrum Sensing in Cognitive Radio: Part II: Multiuser Networks," IEEE Trans. Wireless Communications, vol. 6, no. 6, pp. 2214-2222, 2007.

[16] S.M. Mishra, A. Sahai, and R.W. Broderson, "Cooperative Sensing Among Cognitive Radios," in Proc. IEEE International Conf. Communications, Istanbul, pp. 1658-1663, June 2006.

[17] Y. Zhao., D. Raymond, C. R. C. M. da Silva, J. H. Reed, and S. F. Midkiff, "Performance Evaluation of Radio Environment MapEnabled Cognitive Spectrum-Sharing Networks," in Proc. IEEE Military Comm. Conf., Orlando, FL, pp. 1-7, October 2007.

[18] W. Krenik and A. Batra, "Cognitive Radio Techniques for Wide Area Networks," in Proc. Conference on Design Automation, San Diego, CA, pp. 409-412, June 2005.

[19] W. Krenik and C. Panasik, "The Potential for Unlicensed Wide Area Networks," Wireless Advanced Architectures Group, Texas Instruments White Paper, November 2004.

[20] R. Hachemani, J. Palicot, and C. Moy, "The "Sensorial Radio Bubble" for Cognitive Radio Terminals", International Union of Radio Science (URSI), Chicago, August 2008.

[21] L. Bolea, J. Pérez-Romero, R. Agustí, and O. Sallent, "Primary Transmitter Discovery based on Image Processing in Cognitive Radio," Spring:, EUNICE Conference, Barcelona, pp. 178-187, Sep. 2009.

[22] "Morphological operations", Image Processing Toolbox, The Mathworks. Available at: http://www.mathworks.com/access/helpdesk/help/toolbox/images/i ndex.html, Febr. 2010.

[23] R. Fisher, S. Perkins, A. Walker, and E. Wolfart (2003), "Connected Component Labeling," Available at: http://homepages.inf.ed.ac.uk/rbf/HIPR2/label.htm\#1, Febr. 2010. 